\title{
Evaluation of the predictive indices for candidemia in an adult intensive care unit
}

\section{Gilberto Gambero Gaspar ${ }^{[1]}$, Mayra Gonçalves Menegueti ${ }^{[2]}$, Maria Auxiliadora-Martins ${ }^{[3]}$, Anibal Basile-Filho ${ }^{[3]}$ and Roberto Martinez ${ }^{[1]}$}

[1]. Departamento de Medicina Interna, Faculdade de Medicina de Ribeirão Preto, Universidade de São Paulo, Ribeirão Preto, SP. [2]. Comissão de Controle de Infeccção Hospitalar, Faculdade de Medicina de Ribeirão Preto, Universidade de São Paulo, Ribeirão Preto, SP. [3]. Unidade de Terapia Intensiva, Faculdade de Medicina de Ribeirão Preto, Universidade de São Paulo, Ribeirão Preto, SP.

\begin{abstract}
Introduction: To evaluate predictive indices for candidemia in an adult intensive care unit (ICU) and to propose a new index. Methods: A prospective cohort study was conducted between January 2011 and December 2012. This study was performed in an ICU in a tertiary care hospital at a public university and included 114 patients staying in the adult ICU for at least 48 hours. The association of patient variables with candidemia was analyzed. Results: There were 18 (15.8\%) proven cases of candidemia and $96(84.2 \%)$ cases without candidemia. Univariate analysis revealed the following risk factors: parenteral nutrition, severe sepsis, surgical procedure, dialysis, pancreatitis, acute renal failure, and an APACHE II score higher than 20. For the Candida score index, the odds ratio was 8.50 (95\% CI, 2.57 to 28.09); the sensitivity, specificity, positive predictive value, and negative predictive value were $0.78,0.71,0.33$, and 0.94 , respectively. With respect to the clinical predictor index, the odds ratio was 9.45 ( $95 \% \mathrm{CI}, 2.06$ to 43.39$)$; the sensitivity, specificity, positive predictive value, and negative predictive value were $0.89,0.54,0.27$, and 0.96 , respectively. The proposed candidemia index cutoff was 8.5 ; the sensitivity, specificity, positive predictive value, and negative predictive value were $0.77,0.70,0.33$, and 0.94, respectively. Conclusions: The Candida score and clinical predictor index excluded candidemia satisfactorily. The effectiveness of the candidemia index was comparable to that of the Candida score.
\end{abstract}

Keywords: Candidemia. Candidemia predictors. Risk factors. Bloodstream infection.

\section{INTRODUCTION}

In the United States, the incidence of fungal infections increased 207\% between 1979 and 2000. Candida spp. is the most common causative agent of such infections, accounting for $80 \%$ of cases $^{(1)}$ Candidemia is considered to be the fifth to tenth most common cause of bloodstream infection in patients treated in intensive care units (ICUs) ${ }^{(2)}$.

Colombo et al. ${ }^{(3)}$ conducted a multicenter study in which the authors detected 712 cases of candidemia with an incidence density of 2.49 cases per 1,000 patients per day. Forty-six percent of the patients were staying in the ICU, whereas $39 \%$ were surgical patients. Compared with the United States, the incidence of candidemia in adult patients is higher in Brazil $(0.46 / 1,000$ versus $2.49 / 1,000$, respectively). In both countries, candidemia represents the fourth most frequent cause of bloodstream infection ${ }^{(3)}$

Address to: Dr. Gilberto Gambero Gaspar. Depto. de Clínica Médica/ FMRP/USP. Av. Bandeirantes 3900/sala 1236, Monte Alegre, 14048-500 Ribeirão Preto, SP.

Phone: 5516 3602-2319

e-mail: gilbertogambero@terra.com.br

Received 3 December 2014

Accepted 4 February 2015
Candidemia accounts for high mortality rates, typically when the onset of therapy is delayed for more than 48 hours $^{(4)(5)}$. A positive blood culture for Candida spp. remains the gold standard for the diagnosis of candidemia ${ }^{(6)}$. However, this test cannot detect all cases of candidemia. Additionally, Candida spp. isolation may take too much time, thereby delaying effective antifungal therapy ${ }^{(7)}$. These difficulties have motivated the search for risk factors and candidemia-associated conditions that could help predict this opportunistic infection and design preemptive antifungal therapy.

One study analyzed 2,890 patients who remained in the ICU for at least four days in nine hospitals in the United States and Brazil. The authors proposed a clinical prediction rule for invasive candidiasis in the intensive care setting. The main risk factors were as follows: use of antibiotics, a central catheter, immunosuppressants or corticosteroids; previous surgery; pancreatitis; parenteral nutrition; and dialysis ${ }^{(8)}$.

In 2009, León et al. (9) $^{(2)}$ aluated the use of the Candida score, an index that includes clinical data and multifocal colonization by Candida spp., to discriminate between Candida colonization and candidemia in non-neutropenic critically ill patients. The authors concluded that patients staying at the ICU for more than seven days but with a Candida score lower than 3 were at a $5 \%$ lower risk of candidemia ${ }^{(9)}$.

Posteraro et al. ${ }^{(10)}$ employed the beta-D glucan assay, colonization index, and Candida score to assess 377 patients 
admitted to an ICU. The authors demonstrated that the Candida score provided the second best diagnostic yield: the negative predictive value was high (0.97), but the positive predictive value was low $(0.57)^{(10)}$.

The need for early therapy to treat invasive infection by Candida spp. motivated the present study. We investigated the risk factors for candidemia and evaluated the use of the two mentioned predictors of candidemia in an adult ICU setting in Brazil. In addition, we analyzed a new predictor, the candidemia index.

\section{METHODS}

\section{Clinical setting, design, and study population}

This prospective study aimed to compare a cohort of patients staying at the (ICU) of the University Hospital of the Ribeirão Preto Medical School, University of São Paulo, Ribeirão Preto, SP, Brazil (HC-FMRP-USP) for at least 48 hours. The study was conducted between January 2011 and December 2012.

The following data were collected for the participants: age, origin (clinical or surgical ward), underlying disease, central venous catheter, total parenteral nutrition, use of any type of dialysis, use of immunosuppressants (except corticosteroids), presence of neutropenia $(<500$ neutrophils $/ \mu \mathrm{L})$, previous surgery (any surgical procedure within the previous 30 days), presence of a site colonized by Candida (during ICU stay), intestinal perforation (up to seven days previously), APACHE II (Acute Physiology and Chronic Health Disease Classification System II) score higher than 20, and Enterobacter bacteremia (up to seven days previously).

The blood culture samples were processed on an automated BD $^{\circledR}$ (Becton Dickinson, USA) system. The yeast isolated from the different samples was identified as Candida spp. by means of the standard procedures of a mycological laboratory and through the use of the VITEK $2^{\circledR}$ system (bioMérieux, France). The latter instrument was also employed to evaluate the Candida susceptibility to fluconazole.

The Ethics Committee of HC-FMRP-USP approved this study under Process number HCRP 6915/2010.

\section{Definition of fungal colonization}

Colonization was defined as Candida spp. isolation in some topography without causing disease in the host. We did not use active surveillance, but we evaluated the results obtained from cultures (urine, stool, tracheal secretion, and others) collected by an intensivist.

\section{Calculation of the Candida score}

The Candida score was calculated on the basis of the following score criteria: multifocal colonization (one score), surgery (one score), total parenteral nutrition (one score), and signs of severe sepsis (two scores), which amounted to five (5) scores ${ }^{(11)}$.

\section{Calculation of the clinical predictor index}

The participants were classified as being at positive risk for candidemia when they were receiving systemic antibiotics or had a central venous catheter as well as at least two of the following risk factors: parenteral nutrition (minimum of three days), dialysis (minimum of three days), surgery (within seven days previously), pancreatitis (within seven days previously), corticosteroids (minimum of three days), and any other immunosuppressants (minimum of seven days). All of the patients were classified (positive or negative risk) according to these criteria ${ }^{(8)}$.

\section{Calculation of the candidemia index}

This study evaluated an alternative criterion to predict candidemia using 16 factors assessed: each factor or condition that was present in the patient was awarded a score of one (total $=16$ ). Patients were classified after 48 hours in the ICU. Only one classification was performed per patient according to the inclusion criteria. Patients were excluded if they had stayed at the ICU less than 48 hours or were hematological patients who had not developed neutropenia ( $<500$ neutrophils/uL) during hospitalization. The risk factors analyzed may have first presented in the ICU or in the patient's original ward. More specifically, the following risk factors were analyzed: parenteral nutrition (minimum of three days), surgery (within the previous seven days), severe sepsis (with organ dysfunction), broad-spectrum antibiotics (previous use, up to 30 days, of fourth-generation cephalosporins, carbapenems or glycopeptides), central venous catheter (fitted during hospital stay), immunosuppressants (minimum of seven days), dialysis (minimum of three days), pancreatitis (within the previous seven days), corticosteroids (minimum of three days), acute renal failure (during hospital stay), intestinal perforation (during hospital stay), nosocomial infection (during hospital stay), APACHE score $>20$, Enterobacter bacteremia (during hospital stay), fungal colonization (evaluated during the ICU stay), and neutropenia (during the ICU stay). The receiver operating curves (ROC) was constructed to establish the best cutoff point for this index to predict candidemia.

\section{Statistical analysis}

To verify the association between the variables, the Fisher's exact test was employed. This procedure was conducted using the software SAS ${ }^{8} 9.2$ (Statistical Analysis System, USA) and PROC FREQ. Comparison between the Candida score groups and the candidemia index relied on the Mann-Whitney test for independent samples.

\section{RESULTS}

\section{Participant characteristics}

This study included 114 adult patients with a mean age of 57.8 years. Clinical patients represented $57.9 \%$ of the study population; the mean APACHE II score was 24.3, and the mean death risk was $49 \%$. Blood culture revealed 18 cases of candidemia, whereas 96 patients did not present with this condition (Table 1). The length of stay at the adult ICU was approximately the same for all the participants regardless of invasive infection by Candida, but the mortality was higher $(61.1 \%)$ in the group with candidemia. 
TABLE 1 - Clinical and demographic characteristics of the study population according to the presence or absence of candidemia among patients admitted to the adult intensive care unit.

\begin{tabular}{lcc}
\hline Characteristics & $\begin{array}{c}\text { Proven candidemia } \\
(\mathrm{n}=18)\end{array}$ & $\begin{array}{c}\text { No candidemia } \\
(\mathrm{n}=96)\end{array}$ \\
\hline Age & $\mathrm{n}$ & $\mathrm{n}$ \\
mean & 55.7 & 58.3 \\
maximum & 89 & 94 \\
minimum & 21 & 16 \\
\hline Sex & $\mathrm{n}(\%)$ & $\mathrm{n}(\%)$ \\
female & $7(38.8)$ & $55(57.3)$ \\
male & $11(61.1)$ & $41(42.7)$ \\
\hline Origin & $\mathrm{n}(\%)$ & $\mathrm{n}(\%)$ \\
clinical ward & $11(61.1)$ & $55(57.3)$ \\
surgical ward & $7(38.9)$ & $41(42.7)$ \\
\hline APACHE II & Score & Score \\
mean & 28.0 & 23.6 \\
maximum & 46 & 42 \\
minimum & 21 & 5 \\
\hline APACHE II: & & 0
\end{tabular}

APACHE II: acute physiology and chronic health evaluation II.

With respect to the underlying disease, 11 (61.1\%) patients with candidemia had neoplasia. Six (54.5\%) and five (45.5\%) of the patients with candidemia had hematological and solid organ malignancies, respectively.

\section{Microbiological data}

Blood cultures isolated Candida albicans $(\mathrm{n}=7 ; 36.8 \%)$, Candida tropicalis $(\mathrm{n}=5 ; 26.3 \%)$, Candida glabrata $(\mathrm{n}=3$; $15.7 \%)$, Candida krusei ( $\mathrm{n}=1 ; 5.2 \%)$, Candida parapsilosis $(\mathrm{n}=1 ; 5.2 \%)$, Candida rugosa $(\mathrm{n}=1 ; 5.2 \%)$, and Candida lipolytica $(\mathrm{n}=1 ; 5.2 \%)$.

With respect to sensitivity, all samples of Candida albicans, Candida tropicalis, and Candida parapsilosis were susceptible to fluconazole. However, 7 Candida krusei samples were resistant to fluconazole (63.6\%): 3 urine culture samples, 2 tracheal secretion samples, 1 blood culture sample and 1 catheter tip sample. All of the samples of Candida glabrata were sensitive to fluconazole. The sensitivity and resistence to fluconazole were $94.34 \%$ and $5.66 \%$, respectively.

\section{Analysis of risk factors for candidemia}

Univariate statistical analysis was used to evaluate whether the studied risk factors associated with candidemia were associated with parenteral nutrition $(\mathrm{p}<0.01)$, surgical procedures $(\mathrm{p}=0.01)$, severe sepsis $(\mathrm{p}<0.01)$, dialysis
TABLE 2 - Risk factors for candidemia in patients admitted to the adult intensive care unit.

\begin{tabular}{|c|c|c|c|c|c|}
\hline \multirow[b]{2}{*}{ Risk Factors } & \multicolumn{2}{|c|}{$\begin{array}{l}\text { Proven candidemia } \\
\qquad(\mathrm{n}=18)\end{array}$} & \multicolumn{2}{|c|}{$\begin{array}{l}\text { No candidemia } \\
\quad(\mathrm{n}=96)\end{array}$} & \multirow[b]{2}{*}{ p-value } \\
\hline & $\mathrm{n}$ & $\%$ & $\mathrm{n}$ & $\%$ & \\
\hline Parenteral nutrition & 12 & 66.6 & 21 & 21.9 & $<0.01^{+}$ \\
\hline Surgery & 9 & 50.0 & 45 & 46.9 & $0.01^{+}$ \\
\hline Severe sepsis* & 18 & 100.0 & 52 & 54.2 & $<0.01^{+}$ \\
\hline Previous antibiotics & 18 & 100.0 & 93 & 96.9 & 0.99 \\
\hline Central venous catheter & 18 & 100.0 & 93 & 96.9 & 0.99 \\
\hline Immunosuppressor** & 4 & 22.2 & 10 & 10.4 & 0.23 \\
\hline Dialysis & 13 & 72.2 & 34 & 35.4 & $0.01^{+}$ \\
\hline Pancreatitis & 2 & 11.1 & 0 & 0.0 & $0.02^{+}$ \\
\hline Corticosteroid & 9 & 50.0 & 56 & 58.3 & 0.61 \\
\hline Renal failure & 16 & 88.9 & 47 & 48.1 & $<0.01^{+}$ \\
\hline Neutropenia & 2 & 11.1 & 1 & 1.0 & 0.41 \\
\hline Intestinal perforation & 1 & 5.5 & 7 & 7.3 & 0.99 \\
\hline Hospital infection & 17 & 94.4 & 70 & 72.9 & 0.07 \\
\hline APACHE II > 20 & 18 & 100.0 & 69 & 71.9 & $0,01^{+}$ \\
\hline Enterobacter bacteremia & 5 & 27.8 & 29 & 30.2 & 0.99 \\
\hline Fungal colonization & 11 & 61.1 & 39 & 40.6 & 0.13 \\
\hline
\end{tabular}

APACHE II: acute physiology and chronic health evaluation II. *Defined as sepsis with organ dysfunction. **Excluding the use of corticosteroids. ${ }^{+}$Statistical significance at $\mathrm{p}<0.05$.

$(p=0.01)$, pancreatitis $(p=0.02)$, acute renal failure $(p<0.01)$, or an APACHE II score higher than $20(\mathrm{p}=0.01)$ (Table 2).

\section{Performance of the Candida score and clinical predictor index}

The incidence of invasive candidemia was $0 \%, 13 \%$ (fourcases), $26 \%$ (six cases), $38.8 \%$ (seven cases), and $100 \%$ (one case) in patients with Candida scores of $0,1,2,3,4$, and 5, respectively.

Table 3 lists the application of the Candida score and the clinical predictor index to all of the patients included in the study. The Candida score was statistically significant for the presumptive diagnosis of candidemia $(\mathrm{p}<0.01)$, and the odds ratio was 8.50 [95\% confidence interval (CI)], from 2.57 to 28.09 ); the sensitivity, specificity, positive predictive value, and negative predictive value were $0.78,0.71,0.33$, and 0.94 , respectively.

The clinical predictor index was also statistically significant for the presumptive diagnosis of candidemia $(\mathrm{p}<0.01)$, and the odds ratio was 9.45 ( $95 \% \mathrm{CI}$, from 2.06 to 43.39$)$; the sensitivity, specificity, positive predictive value, and negative predictive value were $0.89,0.54,0.27$, and 0.96 , respectively (Table 3 ).

\section{Performance of candidemia index compared with the Candida score}

The ROC curve (Figure 1) aided the estimation of the best cutoff point, 8.5, for the candidemia index. The sensitivity, 
TABLE 3 - Prediction of candidemia by the Candida score and clinical predictor index in patients admitted to the adult intensive care unit.

\begin{tabular}{|c|c|c|c|c|c|c|}
\hline \multirow[b]{2}{*}{ Evaluated score } & \multicolumn{2}{|c|}{$\begin{array}{l}\text { Proven candidemia } \\
\qquad(\mathrm{n}=18)\end{array}$} & \multicolumn{2}{|c|}{$\begin{array}{l}\text { No candidemia } \\
\quad(\mathrm{n}=96)\end{array}$} & \multirow[b]{2}{*}{ P-value } & \multirow{2}{*}{$\begin{array}{l}\text { Odds ratio } \\
(\mathrm{CI}=95 \%)\end{array}$} \\
\hline & $\mathrm{n}$ & $\%$ & $\mathrm{n}$ & $\%$ & & \\
\hline Candida score $\geq 3$ & 14 & 77.8 & 28 & 29.2 & $\mathrm{P}<0.01^{+}$ & $8.50(2.57 ; 28.09)$ \\
\hline Candida score $<3$ & 4 & 22.2 & 68 & 70.8 & & \\
\hline positive & 16 & 88.9 & 44 & 45.8 & $\mathrm{P}<0.01^{+}$ & $9.45(2.06 ; 43.39)$ \\
\hline negative & 2 & 11.1 & 52 & 54.2 & & \\
\hline
\end{tabular}

CI: confidence interval ${ }^{+}$Statistical significance at $\mathrm{p}<0.05$.

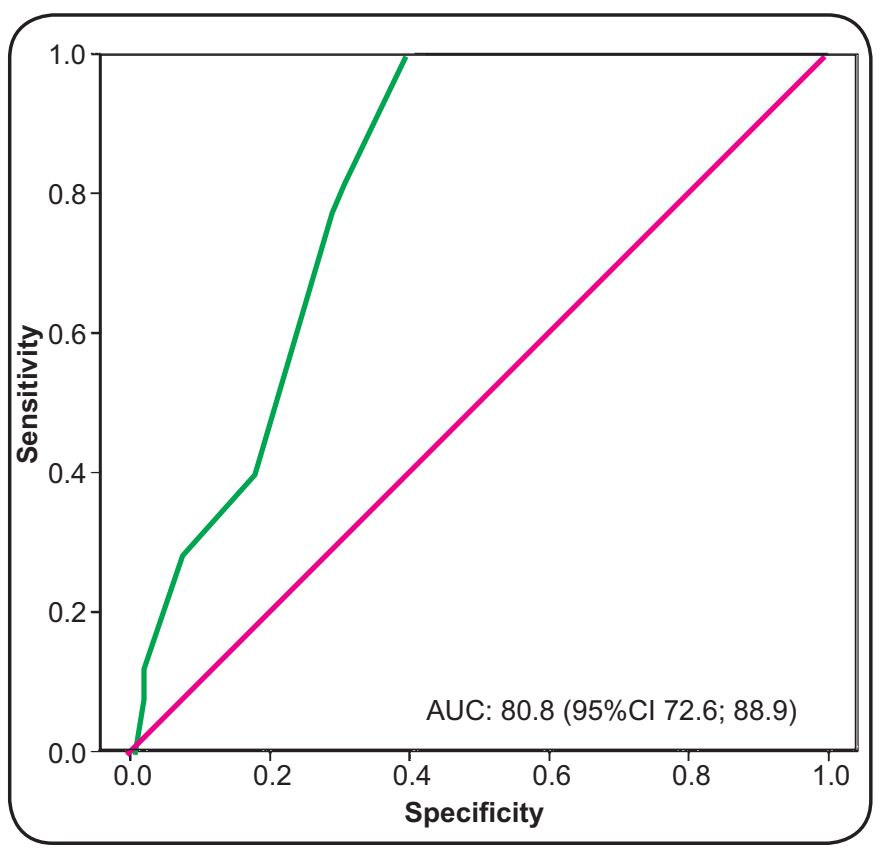

FIGURE 1 - ROC curve for the total score (candidemia index) obtained from the patients according to the number of risk factors for candidemia. AUC: area under the curve; CI: confidence interval; ROC: receiver operating curves. *Value with best sensitivity and specificity.

specificity, positive predictive value, and negative predictive value were $0.77,0.70,0.33$, and 0.94 , respectively. The area under the curve (AUC) was 80.8 , with a $95 \%$ confidence interval $(72.6 ; 88.9)$. The total score statistically correlated $(\mathrm{p}<0.01)$ with cutoff point 3 of the Candida score (Figure 2).

\section{DISCUSSION}

The main achievement of this study was to validate predictive indices that are particularly useful to exclude candidemia in patients admitted to the ICU of a tertiary hospital located in the interior of Brazil.

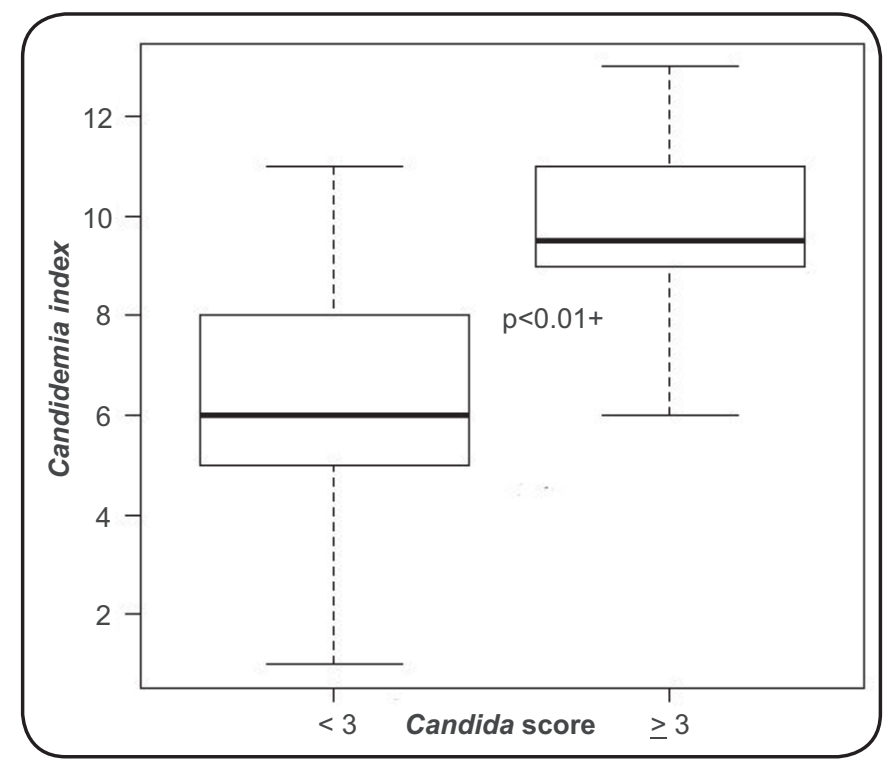

FIGURE 2 - Box plot of the relationship between the total score (candidemia index) and the candida score. ${ }^{+}$Statistical significance at $\mathrm{p}<0.05$.

With respect to severity, the group with candidemia exhibited a higher mean APACHE II score than the group without candidemia. Therefore, patients who acquired candidemia exhibited more critical conditions, which could imply more interventions, such as the frequent use of invasive procedures. Fraser et al. ${ }^{(12)}$ demonstrated that mortality in candidemia patients is directly related to the APACHE II score ${ }^{(12)}$.

In the present study, neoplasia was the most prevalent underlying disease among patients with candidemia. Han et al. ${ }^{(13)}$ reported similar findings, in which candidemia patients had more malignant neoplasms compared to non-candidemia patients $^{(13)}$. When Ostrosky-Zeichner et al. ${ }^{(8)}$ validated the clinical predictor index for candidemia, they also identified neoplasm as a risk factor ${ }^{(8)}$.

Analysis of the patients' progression revealed a mortality of $61.1 \%$ among subjects with candidemia. Various studies have 
also verified a general mean mortality rate and an attributed mortality rate of approximately $60 \%$ and $40 \%$, respectively, among patients with candidemia ${ }^{(9)(14)}$.

Among the risk factors investigated in the present study, we found that parenteral nutrition, severe sepsis, surgical procedures, dialysis, pancreatitis, acute renal failure, and an APACHE II score higher than 20 were associated with candidemia (Table 3). Blumberg et al. ${ }^{(15)}$ identified parenteral nutrition [relative risk $(\mathrm{RR})=3.6$ ], acute renal failure $(R R=4.2)$, and previous surgical procedures $(R R=7.3)$ as risk factors for candidemia ${ }^{(15)}$. Cheng et al. ${ }^{(16)}$ and Mokadas et al. ${ }^{(17)}$ also demonstrated that renal failure was an independent risk factor for the onset of candidemia ${ }^{(16)(17)}$.

In a prospective cohorlt study, León et al. ${ }^{(11)}$ also identified parenteral nutrition, severe sepsis, and multifocal Candida colonization as risk factors ${ }^{(9)(11)}$. In 2009, this same team validated the Candida score and reported a relative risk of 5.9 for the occurrence of candidemia when the total score was equal to or higher than 3. Here, the Candida score provided similar results to those obtained in the validation conducted by those authors ${ }^{(9)(11)}$ : sensitivity 0.78 vs 0.77 , specificity 0.71 vs 0.77 , positive predictive value 0.33 vs 0.13 , and negative predictive value 0.94 vs 0.97 .

In the present study, the incidence of candidemia was low (5\%) when the Candida score was less than 3 and 33\% when the Candida score was equal to or higher than 3. Leroy et al. ${ }^{(18)}$ reported incidences of candidemia of $0 \%, 17.6 \%$, and $50 \%$ in patients with scores of 2 or 3,4 , and 5 , respectively ${ }^{(18)}$. These data confirmed that the Candida score successfully excluded candidemia at the bedside.

Ostrosky-Zeichner et al. ${ }^{(8)}$ described parenteral nutrition, pancreatitis, dialysis, previous surgery, and the use of antimicrobials, central venous catheters, immunosuppressants, or corticosteroids as risk factors for candidemia ${ }^{(8)}$. The clinical predictor index proposed by those authors was evaluated for the patients in the present study and provided increased sensitivity and reduced specificity. The parameters obtained by OstroskyZeichner et al ${ }^{(8)}$ and this study for the clinical predictor index were sensitivity 0.43 vs 0.89 , specificity 0.90 vs 0.54 , positive predictive value 0.01 vs 0.27 , and negative predictive value 0.97 vs 0.96 , respectively.

We assessed a newly proposed index that considered the total score (candidemia index) of each patient included in the study; we adopted a cutoff point of 8.5. In these conditions, the candidemia prediction was similar to that achieved with the Candida score $^{(9)}$. We verified a high correlation between the candidemia index proposed here and the Candida score. The candidemia index must be validated through studies involving a larger number of participants and evaluating different weights for each risk factor.

In conclusions, our data determined that the risk factors associated with candidemia were similar to those observed in hospitals of other geographic regions. The Candida score and the clinical predictor index performed well, which suggested that they might be useful in the clinical setting, especially to exclude candidemia. The candidemia index, a new predictive index for candidemia based on risk factors, was comparable to the Candida score.

\section{ACKNOWLEDGMENTS}

We are grateful for the assistance provided by the Intensive Care Unit staff and the Microbiology Laboratory members working at the University Hospital of the Ribeirão Preto Medical School, University of São Paulo, Ribeirão Preto, Brazil.

\section{CONFLICT OF INTEREST}

The authors declare that there is no conflict of interest.

\section{FINANCIAL SUPPORT}

This study received support from the Fundação de Apoio ao Ensino, Pesquisa e Assistência, (FAEPA) of the University Hospital of the Ribeirão Preto Medical School, University of São Paulo, Ribeirão Preto, Brazil.

\section{REFERENCES}

1. Martin GS, Mannino DM, Eaton S, Moss M. The epidemiology of sepsis in the United States from 1979 through 2000. N Engl J Med 2003; 348:1546-1554.

2. Bouza E, Munoz P. Epidemiology of candidemia in intensive care units. Int J Antimicrob Agents 2008; 32 (suppl 2):87-91.

3. Colombo AL, Nucci M, Park BJ, Nouer SA, Arthington-Skaggs B, Da Matta DA, et al. Epidemiology of candidemia in Brazil: a nationwide sentinel surveillance of candidemia in eleven medical centers. J Clin Microbiol 2006; 44:2816-2823.

4. Nguyen MH, Peacock Jr JE, Tanner DC, Morris AJ, Nguyen ML, Snydman DR, et al. Therapeutic approaches in patients with candidemia. Evaluation in a multicenter, prospective, observational study. Arch Intern Med 1995; 155:2429-2435.

5. Nucci M, Colombo AL, Silveira F, Richtmann R, Salomão R, Branchini ML, et al. Risk factors for death in patients with candidemia. Infect Control Hosp Epidemiol 1998; 19:846-850.

6. Pappas PG, Kauffman CA, Andes D, Benjamin Jr DK, Calandra $\mathrm{TF}$, Edwards Jr JE, et al. Clinical practice guidelines for the management of candidiasis: 2009 update by the Infectious Diseases Society of America. Clin Infect Dis 2009; 48:503-535.

7. Wilson ML, Davis TE, Mirrett S, Reynolds J, Fuller D, Allen SD, et al. Controlled comparison of the BACTEC high-blood-volume fungal medium, BACTEC Plus 26 aerobic blood culture bottle, and 10 -milliliter isolator blood culture system for detection of fungemia and bacteremia. J Clin Microbiol 1993; 31:865-871.

8. Ostrosky-Zeichner L, Sable C, Sobel J, Alexander BD, Donowitz G, Kan V, et al. Multicenter retrospective development and validation of a clinical prediction rule for nosocomial invasive candidiasis in the intensive care setting. Eur J Clin Microbiol Infect Dis 2007; 26:271-276

9. Leon C, Ruiz-Santana S, Saavedra P, Galvan B, Blanco A, Castro C, et al. Usefulness of the "Candida score" for discriminating between Candida colonization and invasive candidiasis in non-neutropenic critically ill patients: a prospective multicenter study. Crit Care Med 2009; 37:1624-1633.

10. Posteraro B, De PG, Tumbarello M, Torelli R, Pennisi MA, Bello G, et al. Early diagnosis of candidemia in intensive care unit patients 
with sepsis: a prospective comparison of (1->3)-beta-D-glucan assay, Candida score, and colonization index. Crit Care 2011; 15:R249.

11. Leon C, Ruiz-Santana S, Saavedra P, Almirante B, Nolla-Salas J, Alvarez-Lerma F, et al. A bedside scoring system ("Candida score") for early antifungal treatment in nonneutropenic critically ill patients with Candida colonization. Crit Care Med 2006; 34:730-737.

12. Fraser VJ, Jones M, Dunkel J, Storfer S, Medoff G, Dunagan WC. Candidemia in a tertiary care hospital: epidemiology, risk factors, and predictors of mortality. Clin Infect Dis 1992; 15:414-421.

13. Han SS, Yim JJ, Yoo CG, Kim YW, Han SK, Shim YS, et al. Clinical characteristics and risk factors for nosocomial candidemia in medical intensive care units: experience in a single hospital in Korea for 6.6 years. J Korean Med Sci 2010; 25:671-676.

14. Pfaller MA. Nosocomial candidiasis: emerging species, reservoirs, and modes of transmission. Clin Infect Dis 1996; 22 (suppl 2):89-94.
15. Blumberg HM, Jarvis WR, Soucie JM, Edwards JE, Patterson JE, Pfaller MA, et al. Risk factors for candidal bloodstream infections in surgical intensive care unit patients: the NEMIS prospective multicenter study. The National Epidemiology of Mycosis Survey. Clin Infect Dis 2001; 33:177-186.

16. Cheng YR, Lin LC, Young TG, Liu CE, Chen CH, Tsay RW. Risk factors for candidemia-related mortality at a medical center in central Taiwan. J Microbiol Immunol Infect 2006; 39:155-161.

17. Mokaddas EM, Al-Sweih NA, Khan ZU. Species distribution and antifungal susceptibility of Candida bloodstream isolates in Kuwait: a 10-year study. J Med Microbiol 2007; 56:255-259.

18. Leroy G, Lambiotte F, Thevenin D, Lemaire C, Parmentier E, Devos P, et al. Evaluation of "Candida score" in critically ill patients: a prospective, multicenter, observational, cohort study. Ann Intensive Care 2011; 1:50. 